\title{
Development and Evaluation of Monoclonal Antibodies for the Glucoside of T-2 Toxin (T2-Glc)
}

\section{Chris M. Maragos ${ }^{1, *}$, Cletus Kurtzman ${ }^{1}$, Mark Busman ${ }^{1}$, Neil Price ${ }^{2}$ and Susan McCormick ${ }^{1}$}

1 Bacterial Foodborne Pathogens and Mycology Research Unit, USDA-ARS-NCAUR, 1815 N. University St., Peoria, IL 61604, USA; E-Mails: cletus.kurtzman@ars.usda.gov (C.K.); mark.busman@ars.usda.gov (M.B.); susan.mccormick@ars.usda.gov (S.M.)

2 Renewable Product Technology Research Unit, USDA-ARS-NCAUR, 1815 N. University St., Peoria, IL 61604, USA; E-Mail: neil.price@ars.usda.gov

* Author to whom correspondence should be addressed; E-Mail: chris.maragos@ars.usda.gov; Tel.: +1-309-681-6266; Fax: +1-309-681-6672.

Received: 5 June 2013; in revised form: 11 July 2013 / Accepted: 12 July 2013 /

Published: 19 July 2013

\begin{abstract}
The interactions between fungi and plants can yield metabolites that are toxic in animal systems. Certain fungi are known to produce sesquiterpenoid trichothecenes, such as T-2 toxin, that are biotransformed by several mechanisms including glucosylation. The glucosylated forms have been found in grain and are of interest as potential reservoirs of T-2 toxin that are not detected by many analytical methods. Hence the glucosides of trichothecenes are often termed "masked" mycotoxins. The glucoside of T-2 toxin (T2-Glc) was linked to keyhole limpet hemocyanin and used to produce antibodies in mice. Ten monoclonal antibody (Mab)-producing hybridoma cell lines were developed. The Mabs were used in immunoassays to detect T2-Glc and T-2 toxin, with midpoints of inhibition curves $\left(\mathrm{IC}_{50} \mathrm{~S}\right)$ in the low $\mathrm{ng} / \mathrm{mL}$ range. Most of the Mabs demonstrated good cross-reactivity to T-2 toxin, with lower recognition of HT-2 toxin. One of the clones (2-13) was further characterized with in-depth cross-reactivity and solvent tolerance studies. Results suggest Mab 2-13 will be useful for the simultaneous detection of T-2 toxin and T2-Glc.
\end{abstract}

Keywords: T-2 toxin; masked mycotoxins; metabolites; antibody; immunoassay 


\section{Introduction}

Various species of fungi routinely cause disease in important cereal crops. Among these, several species of Fusarium infect wheat, maize, oats, barley, and rice. In addition to the loss of value resulting from lowered food quality, the fungi may produce certain secondary metabolites, mycotoxins, which are harmful to animals and humans. T-2 toxin is one of a group of trichothecene mycotoxins produced by $F$. sporotrichioides, F. poae, and F. langsethiae. A related toxin, HT-2 toxin (Figure 1), is believed to be formed by the deacetylation of T-2 toxin during metabolism or bioconversion by microflora.

Figure 1. Structures of certain trichothecene toxins.

\begin{tabular}{llllcc} 
& \\
\hline Common name & $\mathbf{R}^{1}$ & $\mathbf{R}^{2}$ & $\mathbf{R}^{3}$ & $\mathbf{R}^{4}$ & $\mathbf{R}^{5}$ \\
\hline T-2 toxin & ISV & $\mathrm{H}$ & $\mathrm{OAc}^{\text {a }}$ & $\mathrm{OAc}$ & $\mathrm{OH}$ \\
T-2-Glc & $\mathrm{ISV}$ & $\mathrm{H}$ & $\mathrm{OAc}$ & $\mathrm{OAc}$ & $\mathrm{Glc}$ \\
3-Ac-T-2 toxin & $\mathrm{ISV}$ & $\mathrm{H}$ & $\mathrm{OAc}$ & $\mathrm{OAc}$ & $\mathrm{OAc}$ \\
4-deoxy-T-2 & $\mathrm{ISV}$ & $\mathrm{H}$ & $\mathrm{OAc}$ & $\mathrm{H}$ & $\mathrm{OH}$ \\
4-deoxy-T-2-(3-Glc) & $\mathrm{ISV}$ & $\mathrm{H}$ & $\mathrm{OAc}$ & $\mathrm{H}$ & $\mathrm{Glc}$ \\
HT-2 Toxin & $\mathrm{ISV}$ & $\mathrm{H}$ & $\mathrm{OAc}$ & $\mathrm{OH}$ & $\mathrm{OH}$ \\
3-Ac-HT-2 toxin (iso T-2) & $\mathrm{ISV}$ & $\mathrm{H}$ & $\mathrm{OAc}$ & $\mathrm{OH}$ & $\mathrm{OAc}$ \\
T-2 Triol & $\mathrm{ISV}$ & $\mathrm{H}$ & $\mathrm{OH}$ & $\mathrm{OH}$ & $\mathrm{OH}$ \\
T-2 tetraol tetra acetate & $\mathrm{OAc}$ & $\mathrm{H}$ & $\mathrm{OAc}$ & $\mathrm{OAc}$ & $\mathrm{OAc}$ \\
8-Ac-Neosolaniol & $\mathrm{OAc}$ & $\mathrm{H}$ & $\mathrm{OAc}$ & $\mathrm{OAc}$ & $\mathrm{OH}$ \\
Neosolaniol & $\mathrm{OH}$ & $\mathrm{H}$ & $\mathrm{OAc}$ & $\mathrm{OAc}$ & $\mathrm{OH}$ \\
Nivalenol & $=\mathrm{O}$ & $\mathrm{OH}$ & $\mathrm{OH}$ & $\mathrm{OH}$ & $\mathrm{OH}$ \\
Fusarenon-X & $=\mathrm{O}$ & $\mathrm{OH}$ & $\mathrm{OH}$ & $\mathrm{OAc}$ & $\mathrm{OH}$ \\
3,15 di-Ac-NIV & $=\mathrm{O}$ & $\mathrm{OH}$ & $\mathrm{OAc}$ & $\mathrm{OH}$ & $\mathrm{OAc}$ \\
Deoxynivalenol (DON) & $=\mathrm{O}$ & $\mathrm{OH}$ & $\mathrm{OH}$ & $\mathrm{H}$ & $\mathrm{OH}$ \\
Tri-Ac-DON & $=\mathrm{O}$ & $\mathrm{OAc}$ & $\mathrm{OAc}$ & $\mathrm{H}$ & $\mathrm{OAc}$ \\
4, 15-Diacetoxyscirpenol & $\mathrm{H} \mathrm{H}_{2}$ & $\mathrm{H}$ & $\mathrm{OAc}$ & $\mathrm{OAc}$ & $\mathrm{OH}$ \\
\hline & & & \\
\hline
\end{tabular}

${ }^{\text {a }} \mathrm{OAc}$ and ISV represent $-\mathrm{OCOCH}_{3}$ and $-\mathrm{OCOCH}_{2} \mathrm{CH}\left(\mathrm{CH}_{3}\right)_{2}$, respectively.

Both toxins are routinely found in commodities, in particular oats. T-2 toxin is believed to act by inhibiting both protein and DNA synthesis and is acutely toxic to many species, including mammals [1]. While poultry are, in general, more resistant to the effects of mycotoxins they are nevertheless susceptible to oral necrosis, asthenia, inappetence, diarrhea, decreased growth rate, decreased egg production, thinner egg shells, and immune-system toxicity of T-2 toxin [2-4]. Poultry can also exhibit neurological effects following exposure to T-2 toxin [4]. The Joint FAO/WHO Expert Committee on Food Additives (JECFA) and the European Food Safety Authority (EFSA) have 
published opinions on the risks posed by T-2 and HT-2 toxins in foods [2,5]. The need to monitor for trichothecenes in commodities and foods has instigated the development of many different methods for their detection. The literature on analysis of T-2 toxin and related trichothecenes has been recently reviewed [6-8]. Techniques that have been developed to detect T-2 toxin include instrument-intensive methods such as liquid chromatography (LC) with diode array detection [9], labeling and fluorescence detection [10], or mass spectrometric detection [11]. To facilitate sample cleanup or more rapid analysis by immunoassay, numerous antibodies have been developed against T-2 and HT-2 toxins [12-28]. In addition to enzyme-linked immunosorbent assays (ELISAs), immunoassays for T-2 toxin include radioimmunoassay [12,16,20,21], lateral flow test strips [26], fluorescence polarization immunoassay [29], an optical immunoassay [30], and an immunochip [31].

Animals and plants alike have mechanisms to metabolize trichothecene mycotoxins, presumably to render them less toxic or facilitate their elimination. The structure of T-2 toxin (Figure 1) reveals several sites for potential metabolism. In addition to deacetylation, other mechanisms involve deacylation and de-epoxidation. Furthermore the parent toxins and their metabolites may be acted upon by phase II metabolism, yielding a variety of conjugated products. The study of the metabolites of mycotoxin exposure in animals (e.g., biomarkers) is intertwined with the study of metabolites of mycotoxins in the fungi and the host plants. In the latter case the products are known colloquially as "hidden" or "masked" mycotoxins. The term is intended to imply that they are poorly detected, or not detected at all, using conventional methods for measuring the parent toxins. There is considerable interest in the possibility of such metabolites serving as reservoirs of the parent toxins, and a recent issue of the World Mycotoxin Journal focused on this subject [32]. Because of the large number of mycotoxins and possibilities for their metabolism, the number of known masked mycotoxins continues to expand, as such, analytical methods for their detection continue to be developed. A biomarker of exposure in one plant or animal system might be a masked toxin for another system; hence "metabolite", "biomarker", and "masked mycotoxin" are not mutually exclusive. Despite such ambiguities an understanding of the formation of such metabolites and their role as potential reservoirs of toxicity is important.

In the case of the trichothecene mycotoxins, several masked mycotoxins have been reported. These have included the glycosylated derivatives of DON [11,33,34]. In particular the 3-glucoside of DON (DON-3-Glc) has been widely described in the literature. DON-3-Glc is one of the very few masked mycotoxins for which analytical standards are commercially available. Commercial immunoassays developed to detect DON have also been shown to detect the DON-3-Glc to varying degrees [16,35-37]. Recently several reports have appeared describing analogous glucosides and glucuronides in the group A trichothecenes that include T-2 and HT-2 toxins [38-42]. The modification of T-2 toxin by yeasts, yielding T-2 toxin with glucose attached at the C-3 position (e.g., T2-Glc) was recently reported [43]. To facilitate the detection of T2-Glc, we endeavored to develop an antibody capable of interacting with this metabolite. While cross-reaction of antibodies with masked mycotoxins, in particular DON-3-Glc, has been demonstrated [37], to our knowledge ours is the first report of an antibody developed specifically against a masked mycotoxin. 


\section{Results and Discussion}

\subsection{Production of Mabs to T2-Glc}

T2-Glc was conjugated to two proteins, OVA and KLH. The T2G-KLH was used to immunize mice, while the T2G-OVA was used as the immobilized antigen for evaluation of potential antibodies. The attachment of T2-Glc to OVA in the T2G-OVA conjugate was evaluated by ESI-MS. To obtain information on the mass and glycosylation heterogeneity of the intact glycoprotein, mass spectra were first acquired of OVA. The full-scan spectrum was obtained and the resulting deconvoluted spectra displayed a series of mass additions to the molecular weight of OVA with a combination of 162 and $203 \mathrm{Da}$ mass increments, corresponding to hexose and $\mathrm{N}$-acetylglucosamine residues. The only glycosylation site of ovalbumin (Asn-292) has been observed to possess a variety of non-sialylated oligosaccharide structures. The mass and degrees of glycosylation of OVA were consistent with observations recorded by Saba et al. [44]. Similarly, ESI mass spectra were acquired of T2G-OVA. The deconvoluted spectra indicated a positive shift of the envelope of the observed masses of the T2G-OVA from those observed for OVA by approximately $1300 \mathrm{Da}$. Given that conjugation with one T2-Glc would be expected to increase protein mass by $656 \mathrm{Da}$, such a shift suggests that, on the average, two T2-Glc were added to each OVA during the conjugation. As such the T2-OVA was expected to be suitable as an immobilized antigen for evaluation of possible T2-Glc antibodies.

The T2G-KLH conjugate was used to immunize 10 mice, all of which developed a serum response to T2-Glc as measured by competitive indirect (CI)-ELISA. Subsequently, two of the mice were used in hybridoma fusions that yielded a total of 53 positive cultures having antibodies that bound T2-Glc. From these10 clones were subsequently isolated and used to produce sufficient antibody for further evaluation. The Mabs were tested for activity against three toxins (T2-Glc, T-2 toxin, HT-2 toxin) by CI-ELISA (Table 1).

Table 1. Response of 10 Mabs to T2-Glc, T-2 toxin, and HT-2 toxin.

\begin{tabular}{ccccccc}
\hline \multicolumn{5}{c}{$\mathbf{I C}_{\mathbf{5 0}}(\mathbf{n g} / \mathbf{m L})^{\mathbf{a}}$} & & \multicolumn{3}{c}{ Cross-reactivity $(\%)^{\mathbf{b}}$} \\
\hline $\mathrm{Mab}$ & $\mathrm{T} 2-\mathrm{Glc}$ & $\mathrm{T}-2$ toxin & HT-2 toxin & Mab & T-2 toxin & HT-2 toxin \\
\hline $1-2$ & $623 \pm 15$ & $614 \pm 47$ & $>60000$ & $1-2$ & $101 \pm 8$ & $<1.0$ \\
$1-3$ & $8.6 \pm 0.8$ & $6.3 \pm 0.6$ & $43.2 \pm 7.2$ & $1-3$ & $135 \pm 14$ & $19.9 \pm 3.3$ \\
$1-4$ & $13.3 \pm 1.7$ & $13.5 \pm 0.6$ & $243 \pm 22$ & $1-4$ & $99.0 \pm 5.0$ & $5.5 \pm 0.5$ \\
$2-5$ & $13.4 \pm 0.6$ & $14.0 \pm 1.9$ & $33.8 \pm 2.8$ & $2-5$ & $96.3 \pm 13.1$ & $39.8 \pm 3.3$ \\
$2-11$ & $17.6 \pm 0.7$ & $20.2 \pm 1.2$ & $562 \pm 71$ & $2-11$ & $86.9 \pm 5.5$ & $3.1 \pm 0.4$ \\
$2-13$ & $3.5 \pm 0.4$ & $3.8 \pm 0.1$ & $271 \pm 19$ & $2-13$ & $91.6 \pm 4.1$ & $1.3 \pm 0.1$ \\
$2-16$ & $13.6 \pm 0.3$ & $17.4 \pm 1.0$ & $321 \pm 16$ & $2-16$ & $78.6 \pm 4.9$ & $4.3 \pm 0.2$ \\
$2-17$ & $11.3 \pm 0.1$ & $16.7 \pm 1.3$ & $118 \pm 12$ & $2-17$ & $67.5 \pm 5.5$ & $9.5 \pm 0.9$ \\
$2-21$ & $28.2 \pm 0.5$ & $23.1 \pm 1.0$ & $255 \pm 4$ & $2-21$ & $122 \pm 5$ & $11.1 \pm 0.2$ \\
$2-44$ & $7.8 \pm 0.4$ & $8.5 \pm 0.2$ & $28.1 \pm 5.4$ & $2-44$ & $91.3 \pm 2.6$ & $27.7 \pm 5.3$ \\
\hline
\end{tabular}

${ }^{a}$ Concentration of compound that inhibited binding of the antibody by $50 \%$ in CI-ELISA. Values shown were derived from triplicate plates for each antibody and indicate the mean \pm 1 standard deviation; ${ }^{\mathrm{b}}$ Percentage cross reactivity relative to T2-Glc. Cross reaction was calculated as [( $\mathrm{IC}_{50}$ of $\mathrm{T} 2-\mathrm{Glc}_{\mathrm{IC}} \mathrm{IC}_{50}$ of analog $) \times 100 \%$. 
Of the antibodies, the Mab from clone 2-13 was the most sensitive to T2-Glc and was also the most sensitive to T-2 toxin. Interestingly, half of the clones (1-2, 1-4, 2-5, 2-11, 2-13, 2-44) were almost equally cross-reactive to T-2 toxin as they were to T2-Glc. Such antibodies would be good for assays where both metabolites need to be measured. Two of the Mab showed better binding for T-2 toxin than for T2-Glc. However, even in those cases (Mab 1-3, 2-21) the responses to the two toxins were fairly close, with the greatest cross-reactivity being only 135\% (Mab 1-3). Taken in the context of all 10 antibodies this implies that the glucose portion of the immunogen (T2G-KLH) was not a major contributor to antibody binding. It further suggests that the glucose portion of the molecule acted predominantly as a linker between the T-2 toxin and the protein, and was not a focal point for the immune response.

T-2 toxin and HT-2 toxin have similar structures, but differ in that T-2 toxin has an acetate at the C-4 position that is absent in HT-2 toxin (Figure 1). The data in Table 1 reveal that none of the antibodies recognized HT-2 toxin better than T-2 toxin or T2-Glc. Eight of the 10 Mabs demonstrated cross-reactivities to HT-2 toxin of less than 20\% (Table 1). Mab 2-5, with a relative activity of approximately $40 \%$ was the most cross-reactive to HT-2 toxin. The relatively poorer recognition of HT-2 toxin and good recognition of T-2 toxin demonstrated by most of the antibodies, suggests that the acetate at the $\mathrm{C} 4$ position of the trichothecene was an important contributor to the antibody binding and likely an important contributor to the immune response.

\subsection{Cross-Reactivity of Mab 2-13 with Additional Trichothecenes}

From the results in Table 1 it was clear that one of the antibodies (2-13) was more sensitive for T2-Glc and T-2 toxin than the others. In additional assays the Mab 2-13 demonstrated an $\mathrm{IC}_{50}$ of $3.25 \mathrm{ng} / \mathrm{mL}$ of T-2 toxin, which was not significantly different from the $3.5 \mathrm{ng} / \mathrm{mL}$ reported in Table 1 . Because $0.05 \mathrm{~mL}$ was used in each assay, this was equivalent to an $\mathrm{IC}_{50}$ of $162 \mathrm{pg} \mathrm{T}-2$ toxin per assay. There is considerable literature on the development of immunoassays for T-2 toxin [12-28]. Unfortunately not all publications use the same metrics for reporting upon assay sensitivity. However, many reports have given some indication of $\mathrm{IC}_{50}$ either in the form of amount per assay (that is pg T-2 toxin/assay) or concentration (that is $\mathrm{ng} / \mathrm{mL}$ or $\mathrm{pg} / \mathrm{mL}$ ). In certain cases the values have not been reported but can be roughly estimated from dose-response figures within the publication. Of the published immunoassays for T-2 toxin, three appeared notably more sensitive than the others $[22,23,28]$. Remarkably, one assay was reported to have an $\mathrm{IC}_{50}$ of $20 \mathrm{pg} / \mathrm{mL}$ [23]. Many reports have yielded antibodies with similar $\mathrm{IC}_{50} \mathrm{~S}$ as the Mab 2-13 reported here, that is, in the range of $1-5 \mathrm{ng} / \mathrm{mL}$ or circa $0.2 \mathrm{ng}$ /assay [13,15,16,20,25,27]. Assays with sensitivities poorer than these have also been commonly reported. Taken within the context of the existing literature, the CI-ELISA reported here using Mab $2-13$, is neither remarkably better nor worse than previous immunoassays for T-2 toxin. If the Mab 2-13 has an advantage it may be in the ability to bind and detect the T2-Glc. Given our results, it would be of interest to determine if immunoassays previously developed for T-2 toxin might also recognize T2-Glc.

In order to determine which portions of the trichothecene molecule were most important for antibody binding, Mab 2-13 was tested against an additional 14 trichothecenes (Table 2). 
Table 2. Cross-reactivities of 17 trichothecenes with Mab 2-13 in PBS.

\begin{tabular}{lcccccc}
\hline Common name & Cross-Reactivity to Mab 2-13 (\%) & ${ }^{\mathbf{a}} \mathbf{R}^{\mathbf{1}}$ & $\mathbf{R}^{\mathbf{2}}$ & $\mathbf{R}^{\mathbf{3}}$ & $\mathbf{R}^{\mathbf{4}}$ & $\mathbf{R}^{\mathbf{5}}$ \\
\hline T-2-Glc & 100 & ISV & H & OAc & OAc & Glc \\
T-2 toxin & $91.6 \pm 4.1$ & ISV & H & OAc & OAc & OH \\
4-deoxy-T-2-(3-Glc) & $2.6 \pm 0.1$ & ISV & H & OAc & H & Glc \\
8-Ac-Neosolaniol & $2.5 \pm 0.2$ & OAc & H & OAc & OAc & OH \\
3-Ac-T-2 toxin & $2.3 \pm 0.1$ & ISV & H & OAc & OAc & OAc \\
HT-2 Toxin & $1.3 \pm 0.1$ & ISV & H & OAc & OH & OH \\
3-Ac-HT-2 toxin (iso T-2) & 0.1 to 1 & ISV & H & OAc & OH & OAc \\
4-deoxy-T-2 & 0.1 to 1 & ISV & H & OAc & H & OH \\
Neosolaniol & 0.1 to 1 & OH & H & OAc & OAc & OH \\
T-2 Triol & $<0.1$ & ISV & H & OH & OH & OH \\
T-2 tetraol tetra acetate & $<0.1$ & OAc & H & OAc & OAc & OAc \\
Nivalenol (NIV) & $<0.1$ & $=\mathrm{O}$ & OH & OH & OH & OH \\
Fusarenon-X & $<0.1$ & $=\mathrm{O}$ & OH & OH & OAc & OH \\
3,15 di-Ac-NIV & $<0.1$ & $=\mathrm{O}$ & OH & OAc & OH & OAc \\
Deoxynivalenol (DON) & $<0.1$ & $=\mathrm{O}$ & OH & OH & H & OH \\
Tri-Ac-DON & $<0.1$ & $=\mathrm{O}$ & OAc & OAc & H & OAc \\
4,15-Diacetoxyscirpenol & $<0.1$ & H & H & OAc & OAc & OH \\
\hline
\end{tabular}

${ }^{\mathrm{a}} \mathrm{R}$ groups refer to the generic trichothecene structure in Figure 1.

The data suggest that this Mab is highly specific for T-2 toxin and T2-Glc, with poor cross-reactivity for the remaining 15 trichothecenes. However, there were patterns within the data that suggest certain portions of the trichothecene structure were more important to binding than others. An examination of Table 2 reveals that, of the six toxins that cross-reacted more than $1 \%$ with the antibody, only one (8-acetyl-neosolaniol) lacked an isovaleryl group at C-8 (R1 in Figure 1). All six of the most cross-reactive toxins were also reduced at C-7 (R2 in Figure 1) and contained an acetate at C-15 (R3 in Figure 1). The impact of changes to functional groups at positions 8, 7, and 15 on the trichothecene backbone, suggest the importance of this region for recognition by Mab 2-13. However, that was not the only region important for selectivity. As described previously with regard to the cross reactivity of all 10 Mabs to HT-2: the absence of an acetate at position 4 (R4 in Figure 1) significantly reduced binding. The importance of an acetate at position 4 was also revealed by the poor cross-reactivity of 4-deoxy-T2-Glc, which differed from T2-Glc only at R4. The antibody binding site was better able to accommodate certain changes to position 3 (R5 in Figure 1). Substitution of the glucose with a hydroxyl group did not substantially reduce binding, as evidenced by the high cross-reactivity of T-2 toxin. However, substitution with an acetate (e.g., 3-Ac-T2) significantly reduced binding.

These data suggest there may be a certain amount of flexibility allowed for binding at R5. This may be important for future use of the Mab 2-13. The isomer of T2-Glc used to make the immunogen was the same isomer as that produced by yeast cultures (i.e., linked by an axial, or $\alpha$, glycosidic bond). While the $\alpha$-isomer of T2-Glc was naturally produced by the yeast, the identity of the isomers of T2-Glc present in naturally contaminated commodities are unknown. Previous reports with the glucoside of the related trichothecene DON have suggested that when acted upon by UDP-glucosyltransferase from Arabidopsis thaliana, the product is the $\beta$ isomer, that is, 3- $\beta$-D-glucopyranosyl-4-DON [45]. Given 
the presence of at least some flexibility of the antibody for changes to the R5 position, it may bind to the $\beta$ form as well. Unfortunately, the lack of an available standard of $\beta$-linked T2-Glc prevented us from testing it for cross-reactivity. Ultimately it will be important to determine which isomer is present in naturally contaminated grains. The need for further research into the cross-reactivity of antibodies to the glycosylated trichothecenes is further supported by the recent discovery of oligoglycosides of DON [46]. It is not difficult to speculate that similar oligoglycoside conjugates with T-2 toxin may also exist in T-2 contaminated foods.

\subsection{Solvent Tolerance}

Methods of analysis for T-2 toxin generally involve an extraction of the toxin from grains using mixtures of methanol or acetontrile with water. Because of the relatively hydrophobic nature of T-2 toxin such mixtures tend to have a high solvent content. Addition of the glucose (i.e., to T2-Glc) increases the polarity of toxin. For these reasons it was important to test the Mab 2-13 for tolerance to multiple levels of solvents. T2-Glc standards were prepared in aqueous solutions of up to 50\% (by volume) methanol or up to $30 \%$ acetonitrile and tested by CI-ELISA. There was a gradual deterioration in assay performance with increased solvent content (Table 3).

Table 3. Effect of Solvents on the Response of Mab 2-13 to T2-Glc.

\begin{tabular}{ccccc}
\hline Solvent & Solvent concentration $^{\mathbf{a}}$ & $\mathbf{I C}_{\mathbf{5 0}} \mathbf{( n g / \mathbf { m L } )}$ & Relative response (\%) $^{\mathbf{c}}$ & $\boldsymbol{N}^{\mathbf{d}}$ \\
\hline PBS & 0 & $3.3 \pm 0.1$ & $100 \%$ & 12 \\
\hline \multirow{3}{*}{ Methanol } & $5 \%$ & $3.3 \pm 0.2$ & $98 \%$ & 3 \\
& $10 \%$ & $3.7 \pm 0.1$ & $87 \%$ & 3 \\
& $20 \%$ & $4.0 \pm 0.3$ & $81 \%$ & 12 \\
& $30 \%$ & $4.9 \pm 0.5$ & $66 \%$ & 3 \\
Acetonitrile & $50 \%$ & $9.2 \pm 0.9$ & $35 \%$ & 3 \\
& $5 \%$ & $3.7 \pm 0.2$ & $87 \%$ & 3 \\
& $10 \%$ & $4.4 \pm 0.3$ & $73 \%$ & 6 \\
& $15 \%$ & $6.3 \pm 0.2$ & $52 \%$ & 3 \\
& $20 \%$ & $7.4 \pm 0.2$ & $44 \%$ & 3 \\
& $30 \%$ & $14.3 \pm 1.5$ & $23 \%$ & 3 \\
\hline
\end{tabular}

${ }^{a}$ Volume percentage of the solvent used to prepare the standards. Because the standards were mixed $(1+1)$ with Mab in the assays, the concentration of solvent present during the competitive step was one half of the concentrations listed; ${ }^{\mathrm{b}}$ Average $\mathrm{IC}_{50} \pm 1$ standard deviation for T2-Glc; ${ }^{\mathrm{c}}$ Response of T2-Glc in indicated solution relative to T2-Glc in PBS $(100 \%)$. Calculated as $\left(\mathrm{IC}_{50}\right.$ in $\left.\mathrm{PBS}\right) /\left(\mathrm{IC}_{50}\right.$ in solvent solution $) \times 100 \%$;

${ }^{\mathrm{d}}$ Number of ELISA plates used to determine the summary statistics listed.

The solvent concentration at which the $\mathrm{IC}_{50}$ doubled was between $30 \%$ and $50 \%$ for methanol, and approximately $20 \%$ for acetonitrile. Therefore the assay was more easily disrupted with acetonitrile than with methanol. Comparison of solvent tolerance of Mab 2-13 with previous T-2 antibodies is difficult, because most of the previous literature reports did not include such data. Baumgartner et al. [27] developed an assay with an $\mathrm{IC}_{50}$ for $\mathrm{T}-2$ toxin of $3.2 \mathrm{ng} / \mathrm{mL}$, which was very similar in sensitivity to that of our assay using Mab 2-13. That report demonstrated that 10\% methanol shifted the response curve to higher concentrations. The assay could be used at up to $10 \%$ methanol, but at $15 \%$ the antibody 
was not able to work. As with our Mab 2-13, the assays were more sensitive to disruption by acetonitrile than methanol [27]. In another case, using a sensitive competitive direct ELISA for T-2 toxin [25], both acetonitrile and ethanol inhibited binding of toxin-peroxidase conjugate to the immobilized antibody. The effect could be avoided by using solvent concentrations of less than $10 \%$. Another report incorporated $10 \%$ methanol during the cross-reactivity studies [19], implying that the assay was able to tolerate at least that level. To determine whether methanol would impact cross-reactivity of the Mab 2-13 assay, the response to T-2 toxin was also tested in 20\% methanol (Figure 2).

Figure 2. Response of the Mab 2-13 CI-ELISA to T-2 and T2-Glc standard solutions in $20 \%$ methanol. T-2 data were fit with a logistic dose-response curve, T2-Glc data were fit with a Lorentzian Cummulative curve. Data are averages from three plates \pm 1 standard deviation.

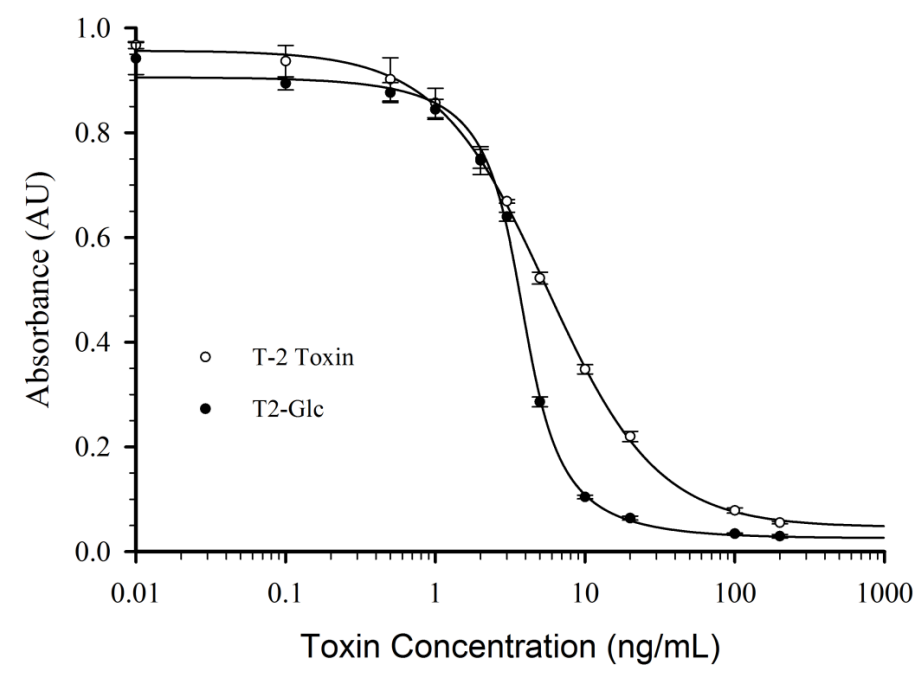

When T-2 toxin was tested in PBS the cross-reactivity was $92 \%$ relative to T2-Glc (e.g., Table 2). However, when T-2 toxin was tested in $20 \%$ methanol, the cross-reactivity was $63 \%$ relative to T2-Glc (Figure 2). This suggests that the relative cross-reactivity will be influenced by the percentage of methanol present. Therefore if Mab 2-13 is to be used to detect T-2 toxin or T2-Glc in commodities, the cross-reactivity should be determined under the solvent conditions specific to such assays. The response curve of T2-Glc in $20 \%$ methanol had a very steep slope between 1 and $10 \mathrm{ng} / \mathrm{mL}$, which may be very useful in an assay based upon signal threshold rather than quantitation. Within the context of these results, and the literature, it appears that the assay with our Mab 2-13 has relatively good solvent tolerance. This, combined with the ability to equally detect T-2 toxin and T2-Glc, may make this antibody useful for the simultaneous detection of T-2 toxin and its 3-glucoside in samples of commodities.

\section{Experimental Section}

\subsection{Reagents}

Chicken egg albumin (OVA), polyvinyl alcohol (PVA, average molecular weight 30,000 to 70,000), and 1,1'-carbonyldiimidazole (CDI) were purchased from Sigma-Aldrich (Milwaukee, WI, USA). Keyhole limpet hemocyanin (KLH) was purchased from Pierce Chemical (Rockford, IL, USA). 
Peroxidase conjugated goat anti-mouse IgG was purchased from Jackson ImmunoResearch Laboratories, Inc. (West Grove, PA, USA). T-2 toxin and HT-2 toxin were obtained from Sigma-Aldrich. The following trichothecenes were prepared at the National Center for Agricultural Utilization Research (NCAUR; Peoria, IL, USA) by one of the co-authors (S.M.) from Fusarium cultures: deoxy-T2, iso-T-2 toxin, 3-Ac-T2, T-2 triol, TTTA, NEO, 8-Ac-NEO, Tri-Ac-DON, FX, 3,15- diAc-NIV, and DAS. T2-Glc and deoxy-T2-Glc were produced at NCAUR by incorporating T-2 toxin or 4-deoxy T-2 toxin into the culture medium for the yeast Blastobotrys muscicola. They were isolated as described previously [43]. Data from NMR indicated that the glucosidyl group was O-linked to the T-2 toxin by an axial $(\alpha-)$ glycosidic bond [43]. Stock solutions of T2-Glc were prepared by gravimetric methods followed by dilution in acetonitrile.

\subsection{HPLC with Photodiode Array Detection}

The purity of the T-2 toxin, HT-2 toxin, and T2-Glc were also assessed by HPLC with photodiode array detection. The instrumentation consisted of a Dionex Ultimate 3000 System (Thermo Fisher, Pittsburgh, PA, USA). Solvent A was acetonitrile, solvent B was water. The column was a Phenomenex Kinetix C-18, $2.6 \mu \mathrm{m}, 4.6 \mathrm{~mm} \times 15 \mathrm{~cm}$, equipped with a Phenomenex RP guard cartridge. The mobile phase was a gradient, with solvent $\mathrm{A}$ acetonitrile and solvent $\mathrm{B}$ water, as follows: equilibrate for 3.5 min with $30 \% \mathrm{~A}$; inject sample; linear ramp from $30 \% \mathrm{~A}$ to $50 \% \mathrm{~A}$ over 6 min; linear ramp to $90 \% \mathrm{~A}$ over $1.5 \mathrm{~min}$; hold at $90 \% \mathrm{~A}$ for $1.5 \mathrm{~min}$; then return to the equilibration condition at the end of the run (e.g., at $9 \mathrm{~min}$ ). Flow rate of $1.7 \mathrm{~mL} / \mathrm{min}$. The detector was programmed to scan the range from 190 to $300 \mathrm{~nm}$, with monitoring at $202 \mathrm{~nm}$, data collection rate $10 \mathrm{~Hz}$. The volumes injected were $10 \mu \mathrm{L}$. A sample chromatogram is indicated in Figure 3.

Figure 3. HPLC chromatogram of T2-Glc used to prepare the protein conjugates. The arrows indicate retention times for T2-Glc (3.17 $\mathrm{min})$, HT-2 toxin $(3.30 \mathrm{~min})$, and T-2 toxin $(5.35 \mathrm{~min})$. The amount of T2-Glc injected was $250 \mathrm{ng}$.

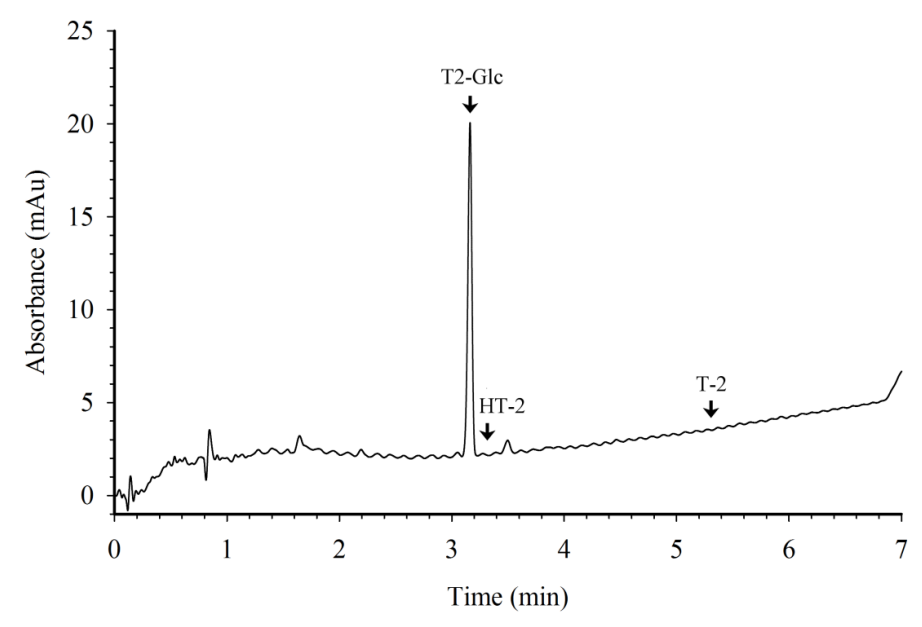

\subsection{Preparation and Evaluation of T2-Glc Protein Conjugates}

Protein conjugates of T2-Glc were synthesized by linking the hydroxyl groups of the toxin to the primary amines of the proteins using a carbodiimide technique similar to that described previously for 
DON [47]. The immunogen was a conjugate of T2-Glc with KLH (T2G-KLH). On the day of the reaction $4 \mathrm{mg}$ of T2-Glc was dissolved in $0.4 \mathrm{~mL}$ acetone, and $75 \mathrm{mg}$ of CDI was added. The vessel was sealed and held at ambient temperature for $1 \mathrm{~h}$, after which $0.05 \mathrm{~mL}$ of water was added, followed by $1 \mathrm{~mL}$ of KLH solution ( $20 \mathrm{mg}$ in $0.1 \mathrm{M}$ sodium bicarbonate buffer, $\mathrm{pH} 8.5$ ). The mixture was incubated for $24 \mathrm{~h}$ at $4{ }^{\circ} \mathrm{C}$ and then dialyzed against five sequential changes of PBS to remove unbound T2-Glc. The T2G-KLH was diluted to $2 \mathrm{mg} / \mathrm{mL}$ with $0.1 \mathrm{M} \mathrm{PBS}$, then freeze-dried and sent to Harlan Bioproducts for Science (Madison, Wisconsin, USA) for administration into mice. The test antigen, a conjugate of T2-Glc with ovalbumin (T2G-OVA) was prepared in a similar fashion. The T2G-OVA was evaluated by mass spectrometry to determine the degree of conjugation with T2-Glc. The mass spectrometer (MS) used was an Exactive-MS (Thermo Fisher Scientific, Waltham, MA, USA) equipped with an electrospray ionization (ESI) source. For all experiments the MS was operated in positive ionization mode. Samples were sprayed using a $4.00 \mathrm{kV}$ needle voltage, and optimized parameters of the system for MS detection were: inlet capillary voltage: $+90.0 \mathrm{~V}$; tube lens voltage: $+190 \mathrm{~V}$; capillary temperature: $275 \mathrm{C}$; skimmer voltage: $+40.0 \mathrm{~V}$. All analyses were conducted with an automated gain control setting of $5 \times 10^{5}$, a resolution setting of 100,000 FWHM, a scan speed of $1 \mathrm{~Hz}$ and a mass scan range of 500-3000 Da. Aqueous solutions of OVA or T2G-OVA (1 mg/mL) were infused into the ESI source with a syringe pump at a flow rate of $5 \mu \mathrm{L} / \mathrm{min}$. A $20 \mu \mathrm{L} / \mathrm{min}$ supplemental flow of $1+1$ $(v / v)$ methanol/water with $2 \%$ acetic acid, provided by a high performance liquid chromatography pump, was added to the syringe pump flow via a tee immediately prior to the ESI source.

\subsection{Immunizations and Screening for T2-Glc Specific Antibodies by CI-ELISA}

Immunization of mice and collection of sera were conducted at Harlan Bioproducts for Science (Madison, WI, USA). Ten female Balb/C mice were initially immunized by injection of $100 \mu \mathrm{g}$ T2G-KLH per animal using the same procedures as described previously for production of fusarin antibodies [48]. A CI-ELISA was developed and used for screening of mouse sera and culture supernatants for the presence of antibodies. Each hybridoma culture supernant solution was tested in single replicates at a minimum of three dilutions, and positive cultures were re-assayed. For screening assays $0.1 \mathrm{ml}$ of T2G-OVA, $1 \mu \mathrm{g} / \mathrm{mL}$ in $0.05 \mathrm{M}$ sodium phosphate buffer ( $\mathrm{pH} 7$ ), was added to wells of polystyrene microtiter plates and allowed to attach overnight at $4{ }^{\circ} \mathrm{C}$. After washing the coated plate twice with $0.32 \mathrm{~mL}$ Tween-PBS (0.02\% v/v Tween-20 in 0.01 M PBS pH 7), $0.32 \mathrm{~mL}$ of PVA-PBS (1\% $w / v$ PVA in $0.01 \mathrm{M}$ PBS) was added and allowed to incubate at ambient temperature for $2 \mathrm{~h}$. During this incubation, test solutions were prepared. The test solutions consisted of $0.06 \mathrm{~mL}$ of toxin standard solutions (or PBS control) mixed with $0.06 \mathrm{~mL}$ of serum (or culture fluid) diluted in BSA-PBS $(1 \% w / v$ BSA in $0.01 \mathrm{M} \mathrm{PBS})$ in the wells of a polypropylene microwell plate (Corning Inc., Corning, NY, USA). The wells of the polystyrene (T2G-OVA coated) plate were washed twice with Tween-PBS and $0.1 \mathrm{~mL}$ of test solution was transferred into each well. After incubation at ambient temperature for $30 \mathrm{~min}$ the plate was washed three times and $0.1 \mathrm{~mL}$ of goat anti-mouse peroxidase conjugate (diluted 1:2000 in BSA-PBS) was added. The plate was incubated for 30 min at ambient temperature then washed four times before addition of $0.1 \mathrm{~mL}$ of the $O$-phenylenediamine (OPD) substrate. The OPD solution was prepared by combining $0.02 \mathrm{~mL}$ of $30 \% \mathrm{H}_{2} \mathrm{O}_{2}$ and $20 \mathrm{mg}$ OPD in $50 \mathrm{~mL}$ of citrate-phosphate buffer $(0.05 \mathrm{M}$ citrate, $0.1 \mathrm{M}$ phosphate, $\mathrm{pH} 5.0)$. After $5 \mathrm{~min}$ at 
ambient temperature, the reaction was stopped by the addition of $0.1 \mathrm{~mL}$ of $1 \mathrm{~N}$ hydrochloric acid. Color development was determined by measuring the absorbance at $490 \mathrm{~nm}$ using a Synergy HT microplate reader (Bio-Tek, Winooski, VT, USA).

\subsection{Production and Purification of Monoclonal Antibodies}

Two mice having sera with antibodies reactive to T2-Glc were sacrificed and aseptically splenectomized at Harlan Bioproducts for Science (Madison, WI, USA). Spleenocytes were chemically fused with Balb/C non-immunoglobulin secreting (NS-1) myeloma cells using polyethylene glycol. Fused cells were plated in HAT selection media. After 11 days, HAT resistant cultures were isolated and screened for anti-T2-Glc activity by CI-ELISA. The two fusions yielded a total of 53 positive cultures. From these 10 cultures were subsequently cloned, expanded and frozen. The cultures are named here as: $1-2,1-3,1-4,2-5,2-11,2-13,2-16,2-17,2-21$, and 2-44. With this nomenclature the first digit describes the fusion and the second digit describes the hybridoma cell line derived from that fusion. All antibodies were isotype $\operatorname{IgG}_{1}$. The 10 cell lines were expanded to produce ascites fluid in mice using established procedures [49]. The ascites fluid was partially purified by ammonium sulfate precipitation using procedures described previously [47], then lyophilized. Protein content of each of the preparations was determined using the BCA Protein Assay according to the protocols provided by the manufacturer (Thermo Fisher, Pittsburgh, PA, USA).

\subsection{Cross Reactivity of Mabs and Solvent Effects}

The ability of isolated Mabs to cross-react with related trichothecenes was tested by CI-ELISA. Standard T2-Glc or other trichothecene mycotoxins were solubilized and diluted to $1 \mathrm{mg} / \mathrm{mL}$ with either acetonitrile or methanol. These stocks were further diluted with PBS to produce standard solutions with concentrations from 0.1 to $10,000 \mathrm{ng} / \mathrm{mL}$. Preliminary experiments were used to establish narrower concentration ranges that were then used to determine the concentrations causing $50 \%$ inhibition of signal $\left(\mathrm{IC}_{50} \mathrm{~s}\right)$. The CI-ELISA protocol was the same as that described for screening of the cultures, with the exception that a lower level of T2G-OVA $(0.4 \mu \mathrm{g} / \mathrm{mL})$ was immobilized. Because each Mab preparation contained different levels of protein, preliminary experiments were used to determine the concentrations of each of the antibodies that would provide optimal signal in the assays (an absorbance of 0.8 to 1.1 for toxin-free controls). The cell lines and corresponding protein concentrations added in the cross-reactivity tests were: 1-2 $(2 \mu \mathrm{g} / \mathrm{mL}), 1-3(4 \mu \mathrm{g} / \mathrm{mL}), 1-4(4 \mu \mathrm{g} / \mathrm{mL})$, 2-5 (4 $\mu \mathrm{g} / \mathrm{mL}), 2-11(8 \mu \mathrm{g} / \mathrm{mL}), 2-13(2 \mu \mathrm{g} / \mathrm{mL}), 2-16(4 \mu \mathrm{g} / \mathrm{mL}), 2-17(4 \mu \mathrm{g} / \mathrm{mL}), 2-21(10 \mu \mathrm{g} / \mathrm{mL})$, and 2-44 $(2 \mu \mathrm{g} / \mathrm{mL})$. All $10 \mathrm{Mab}$ were tested for cross-reactivity to T2-Glc, T-2 toxin, and HT-2 toxin. Based upon those results, further experiments were conducted to determine cross-reactivity of Mab 2-13 to 14 additional trichothecenes.

\section{Conclusions}

Ten Mab-producing hybridoma cell lines were developed that recognized the masked mycotoxin T2-Glc as well as the parent toxin, T-2 toxin. When used in a CI-ELISA format, most of these Mabs resulted in assays with $\mathrm{IC}_{50} \mathrm{~S}$ in the low $\mathrm{ng} / \mathrm{mL}$ range, with lower cross-reactivity to HT-2 toxin. Mab 
from clone 2-13 was further characterized with additional cross-reactivity and solvent tolerance studies. This Mab was highly specific for T-2 toxin and T2-Glc. The region of the molecule encompassed by positions 8, 7, 15, and 4 (R1 thru R4 in Figure 1) was important for recognition. There was some flexibility in binding at position 3 (R5 in Figure 1), which was also the site of the glucose attachment. The assay with Mab 2-13 demonstrated relatively good solvent tolerance. This, combined with the ability to equally detect T-2 toxin and T2-Glc, may make this antibody useful for the simultaneous detection of both toxins in commodities.

\section{Acknowledgments}

The authors thank Candace Lindgren, Jenny Wiersma, Julie Havlicek, Deanna Morrison, Paula Dodge, Nahla Elmahdy, and Shannon Snodgrass of Harlan Bioproducts for Science, for the exceptional quality of the technical assistance provided throughout this project.

\section{Disclaimer}

Mention of trade names or commercial products in this article is solely for the purpose of providing specific information and does not imply recommendation or endorsement by the U.S. Department of Agriculture. USDA is an equal opportunity provider and employer.

\section{Conflict of Interest}

The authors declare no conflict of interest

\section{References}

1. Ueno, Y. Toxicological features of T-2 toxin and related trichothecenes. Toxicol. Sci. 1984, 4, S124-S132.

2. Canady, R.A.; Coker, R.D.; Egan, S.K.; Krska, R.; Olsen, M.; Resnik, S.; Schlatter, J. T-2 and HT-2 Toxins. In WHO Food Additive Series: 47 Safety Evaluation of Certain Mycotoxins in Food; Joint FAO/WHO Expert Committee on Food Additives (JECFA), FAO Food and nutrition paper 74. 2001. Available online: http://www.inchem.org/documents/jecfa/jecmono/v47je06.htm (accessed on 31 May 2013).

3. Kubena, L.F.; Edrington, T.S.; Harvey, R.B.; Buckley, S.A.; Phillips, T.D.; Rottinghaus, G.E.; Casper, H.H. Individual and combined effects of fumonisin $\mathrm{B}_{1}$ present in Fusarium moniliforme culture material and T-2 toxin or deoxynivalenol in broiler chicks. Poult. Sci. 1997, 76, 1239-1247.

4. Wyatt, R.D. Biological Effects of Mycotoxins (other than Aflatoxin) on Poultry. In Interactions of Mycotoxins in Animal Production; Naber, E.C., Wallace, H.D., Eds.; National Academy of Sciences: Washington, DC, USA, 1979; pp. 87-95.

5. European Food Safety Authority (EFSA) Panel on Contaminants in the Food Chain. Scientific opinion on the risks for animal and public health related to the presence of T-2 and HT-2 toxin in food and feed. EFSA J. 2011, 9, 2481-2667. 
6. Shephard, G.S.; Berthiller, F.; Burdaspal, P.A.; Crews, C.; Jonker, M.A.; Krska, R.; MacDonald, S.; Malone, R.J.; Maragos, C.; Sabino, M.; et al. Developments in mycotoxin analysis: An update for 2010-2011. World Mycotoxin J. 2012, 5, 3-30.

7. Li, Y.; Wang, Z.; Beier, R.C.; Shen, J.; de Smet, D.; de Saeger, S.; Zhang, S. T-2 toxin, a trichothecene mycotoxin: Review of toxicity, metabolism, and analytical methods. J. Agric. Food Chem. 2011, 59, 3441-3453.

8. Meneely, J.P.; Ricci, F.; van Egmond, H.P.; Elliott, C.T. Current methods of analysis for the determination of trichothecene mycotoxins in food. Trends Anal. Chem. 2011, 30, 192-203.

9. Pascale, M.; Panzarini, G.; Visconti, A. Determination of HT-2 and T-2 toxins in oats and wheat by ultra-performance liquid chromatography with photodiode array detection. Talanta 2012, 89, 231-236.

10. Lippolis, V.; Pascale, M.; Maragos, C.M.; Visconti, A. Improvement of detection sensitivity of T-2 and HT-2 toxins using different fluorescent labeling reagents by high-performance liquid chromatography. Talanta 2008, 74, 1476-1483.

11. Rasmussen, P.H.; Nielsen, K.F.; Ghorbani, F.; Spliid, N.H.; Nielsen, G.C.; Jørgensen, L.N. Occurrence of different trichothecenes and deoxynivalenol-3- $\beta$-D-glucoside in naturally and artificially contaminated Danish cereal grains and whole maize plants. Mycotoxin Res. 2012, 28, 181-190.

12. Chu, F.S.; Grossman, S.; Wei, R.D.; Mirocha, C.J. Production of antibody against T-2 toxin. Appl. Environ. Microbiol. 1979, 37, 104-108.

13. Pestka, J.J.; Lee, S.C.; Lau, H.P.; Chu, F.S. Enzyme-linked immunosorbent assay for T-2 toxin. J. Am. Oil Chem. Soc. 1981, 58, A940-A944.

14. Hunter, K.W.; Brimfield, A.A.; Miller, M.; Finkelman, F.D.; Chu, S.F. Preparation and characterization of monoclonal antibodies to the trichothecene mycotoxin T-2. Appl. Environ. Microbiol. 1985, 49, 168-172.

15. Ohtani, K.; Kawamura, O.; Kajii, H.; Chiba, J.; Ueno, Y. Development of enzyme-linked immunosorbent assay (ELISA) for T-2 toxin using monoclonal antibodies. Mycotoxins 1985, 22, $31-32$.

16. Zhang, G.S.; Schubring, S.L.; Chu, F.S. Improved method for production of antibodies against T-2 toxin and diacetoxyscirpenol in rabbits. Appl. Environ. Microbiol. 1986, 51, 132-137.

17. Fan, T.S.; Zhang, G.S.; Chu, F.S. Production and characterization of antibodies against HT-2 toxin and T-2 tetraol tetraacetate. Appl. Environ. Microbiol. 1987, 53, 17-21.

18. Goodbrand, I.A.; Stimson, W.H.; Smith, I.E. A monoclonal antibody to T-2 toxin. Lett. Appl. Microbiol. 1987, 5, 97-99.

19. Gendloff, E.H.; Pestka, J.J.; Dixon, D.E.; Hart, L.P. Production of a monoclonal antibody to T-2 toxin with strong cross-reactivity to T-2 metabolites. Phytopathology 1987, 77, 57-59.

20. Wei, R.D.; Swanson, S.; Chu, F.S. Cross-reactivity of antibodies against T-2 with deepoxide T-2 toxin. Mycotoxin Res. 1988, 4, 15-19.

21. Fan, T.S.L.; Schubring, S.L.; Wei, R.D.; Chu, F.S. Production and characterization of a monoclonal antibody cross-reactive with most group A trichothecenes. Appl. Environ. Microbiol. 1988, 54, 2959-2963. 
22. Chiba, J.; Kawamura, O.; Kajii, H.; Ohtani, K.; Nagayama, S.; Ueno, Y. A sensitive enzyme-linked immunosorbent assay for detection of T-2 toxin with monoclonal antibodies. Food Addit. Contam. 1988, 5, 629-639.

23. Hack, R.; Martlbauer, E.; Terplan, G. A monoclonal antibody-based enzyme immunoassay for the detection of T-2 toxin at picogram levels. Lett. Appl. Microbiol. 1989, 9, 133-135.

24. Chanh, T.C.; Hewetson, J.F. Structure/function studies of T-2 mycotoxin with a monoclonal antibody. Immunopharmacology 1991, 21, 83-89.

25. Barna-Vetró, I.; Gyöngyösi, A.; Solti, L. Monoclonal antibody-based enzyme-linked immunosorbent assay of Fusarium T-2 and zearalenone toxins in cereals. Appl. Environ. Microbiol. 1994, 60, 729-731.

26. Molinelli, A.; Grossalber, K.; Führer, M.; Baumgartner, S.; Sulyok, M.; Krska, R. Development of qualitative and semiquantitative immunoassay-based rapid strip tests for the detection of T-2 toxin in wheat and oat. J. Agric. Food Chem. 2008, 56, 2589-2594.

27. Baumgartner, S.; Führer, M.; Krska, R. Comparison of monoclonal antibody performance characteristics for the detection of two representatives of A- and B-trichothecenes: T-2 toxin and deoxynivalenol. World Mycotoxin J. 2010, 3, 233-238.

28. Wang, J.; Duan, S.; Zhang, Y.; Wang, S. Enzyme-linked immunosorbent assay for the determination of T-2 toxin in cereals and feedstuff. Microchim. Acta 2010, 169, 137-144.

29. Lippolis, V.; Pascale, M.; Valenzano, S.; Pluchinotta, V.; Baumgartner, S.; Krska, R.; Visconti, A. A rapid fluorescence polarization immunoassay for the determination of T-2 and HT-2 toxins in wheat. Anal. Bioanal. Chem. 2011, 401, 2561-2571.

30. Meneely, J.P.; Sulyok, M.; Baumgartner, S.; Krska, R.; Elliott, C.T. A rapid optical immunoassay for the screening of T-2 and HT-2 toxin in cereals and maize-based baby food. Talanta 2010, 81, 630-636.

31. Wang, Y.; Liu, N.; Ning, B.; Liu, M.; Lv, Z.; Sun, Z.; Peng, Y.; Chen, C.; Li, J.; Gao, Z. Simultaneous and rapid detection of six different mycotoxins using an immunochip. Biosens. Bioelectron. 2012, 34, 44-50.

32. De Saeger, S.; van Egmond, H. Special issue: Masked mycotoxins. World Mycotoxin J. 2012, 5, 203-206.

33. Zachariasova, M.; Hajslova, J.; Kostelanska, M.; Poustka, J.; Krplova, A.; Cuhra, P.; Hochel, I. Deoxynivalenol and its conjugates in beer: A critical assessment of data obtained by enzyme-linked immunosorbent assay and liquid chromatography coupled to tandem mass spectrometry. Anal. Chim. Acta 2008, 625, 77-86.

34. Berthiller, F.; Schuhmacher, R.; Adam, G.; Krska, R. Formation, determination and significance of masked and other conjugated mycotoxins. Anal. Bioanal. Chem. 2009, 395, 1243-1252.

35. Tangni, E.K.; Motte, J.C.; Callebaut, A.; Pussemier, L. Cross-reactivity of antibodies in some commercial deoxynivalenol test kits against some fusariotoxins. J. Agric. Food Chem. 2010, 58, $12625-12633$.

36. Versilovskis, A.; Huybrecht, B.; Tangni, E.K.; Pussemier, L.; de Saeger, S.; Callebaut, A. Cross-reactivity of some commercially available deoxynivalenol (DON) and zearalenone (ZEN) immunoaffinity columns to DON- and ZEN-conjugated forms and metabolites. Food Addit. Contam. Part A 2011, 28, 1687-1693. 
37. Goryacheva, I.Y.; de Saeger, S. Immunochemical detection of masked mycotoxins: A short review. World Mycotoxin J. 2012, 5, 281-287.

38. Busman, M.; Poling, S.M.; Maragos, C.M. Observation of T-2 toxin and HT-2 toxin glucosides from Fusarium sporotrichioides by liquid chromatography coupled to tandem mass spectrometry (LC-MS/MS). Toxins 2011, 3, 1554-1568.

39. Lattanzio, V.M.T.; Visconti, A.; Haidukowski, M.; Pascale, M. Identification and characterization of new Fusarium masked mycotoxins, T2 and HT2 glycosyl derivatives, in naturally contaminated wheat and oats by liquid chromatography high-resolution mass spectrometry. J. Mass. Spectrom. 2012, 47, 466-475.

40. Veprikova, Z.; Vaclavikova, M.; Lacina, O.; Dzuman, Z.; Zachariasova, M.; Hajslova, J. Occurrence of mono- and di-glycosylated conjugates of T-2 and HT-2 toxins in naturally contaminated cereals. World Mycotoxin J. 2012, 5, 231-240.

41. Nakagawa, H.; Ohmichi, K.; Sakamoto, S.; Sago, Y.; Kushiro, M.; Nagashima, H.; Yoshida, M.; Nakajima, T. Detection of a new Fusarium masked mycotoxin in wheat grain by high-resolution LC-Orbitrap MS. Food Addit. Contam. Part A 2011, 28, 1447-1456.

42. Nakagawa, H.; Sakamoto, S.; Sago, Y.; Kushiro, M.; Nagashima, H. The use of LC-Orbitrap MS for the detection of Fusarium masked mycotoxins: the case of type A trichothecenes. World Mycotoxin J. 2012, 5, 271-280.

43. McCormick, S.P.; Price, N.P.J.; Kurtzman, C.P. Glucosylation and other biotransformations of T-2 toxin by yeasts of the Trichomonascus clade. Appl. Environ. Microbiol. 2012, 78, 8694-8702.

44. Saba, J.A.; Shen, X.; Jamieson, J.C.; Perreault, H. Investigation of different combinations of derivitization, separation methods and electrospray ionization mass spectrometry for standard oligosaccharides and glycans from ovalbumin, J. Mass Spectrom. 2001, 36, 563-574.

45. Poppenberger, B.; Berthiller, F.; Lucyshyn, D.; Sieberer, T.; Schuhmacher, R.; Krska, R.; Kuchler, K.; Glössl, J.; Luschnig, C.; Adam, G. Detoxification of Fusarium mycotoxin deoxynivalenol by a UDP-glucosyltransferase from Arabidopsis thaliana. J. Biol. Chem. 2003, 278, 47905-47914.

46. Zachariasova, M.; Vaclavikova, M.; Lacina, O.; Vaclavik, L.; Hajslova, J. Deoxynivalenol oligoglycosides: New "masked" Fusarium toxins occurring in malt, beer, and breadstuff. J. Agric. Food Chem. 2012, 60, 9280-9291.

47. Maragos, C.M.; McCormick, S.P. Monoclonal antibodies for the mycotoxins deoxynivalenol and 3-acetyl-deoxynivalenol. Food Agric. Immunol. 2000, 12, 181-192.

48. Maragos, C.M.; Busman, M.; Plattner, R.D. Development of monoclonal antibodies for the fusarin mycotoxins. Food Addit. Contam. 2008, 25, 105-114.

49. Hoogenraad, N.; Newman, T.; Hoogenraad, J. The effect of pre-injection of mice with pristine on ascites tumour formation and monoclonal antibody production. J. Immunol. Methods 1983, 61, $317-320$.

(C) The authors are employees of the U.S. Department of Agriculture (USDA), and the article is a U.S. Government Work, published by MDPI, Basel, Switzerland, with the permission of USDA. This article is an open access article distributed under the terms and conditions of the Creative Commons Attribution license (http://creativecommons.org/licenses/by/3.0/). 